\section{Infected Implantable Pulse Generator}

The Neurohospitalist 2019, Vol. 9(3) 172-173

(C) The Author(s) 2018

Article reuse guidelines:

sagepub.com/journals-permissions

DOI: $10.1177 / 19418744 \mid 880986$

journals.sagepub.com/home/ $\mathrm{NHO}$

\author{
Shefali Dujari, MD'® and Carl A. Gold, MD, MS'
}

(S)AGE

\title{
Keywords
}

functional imaging, infectious disease medicine, Parkinson disease

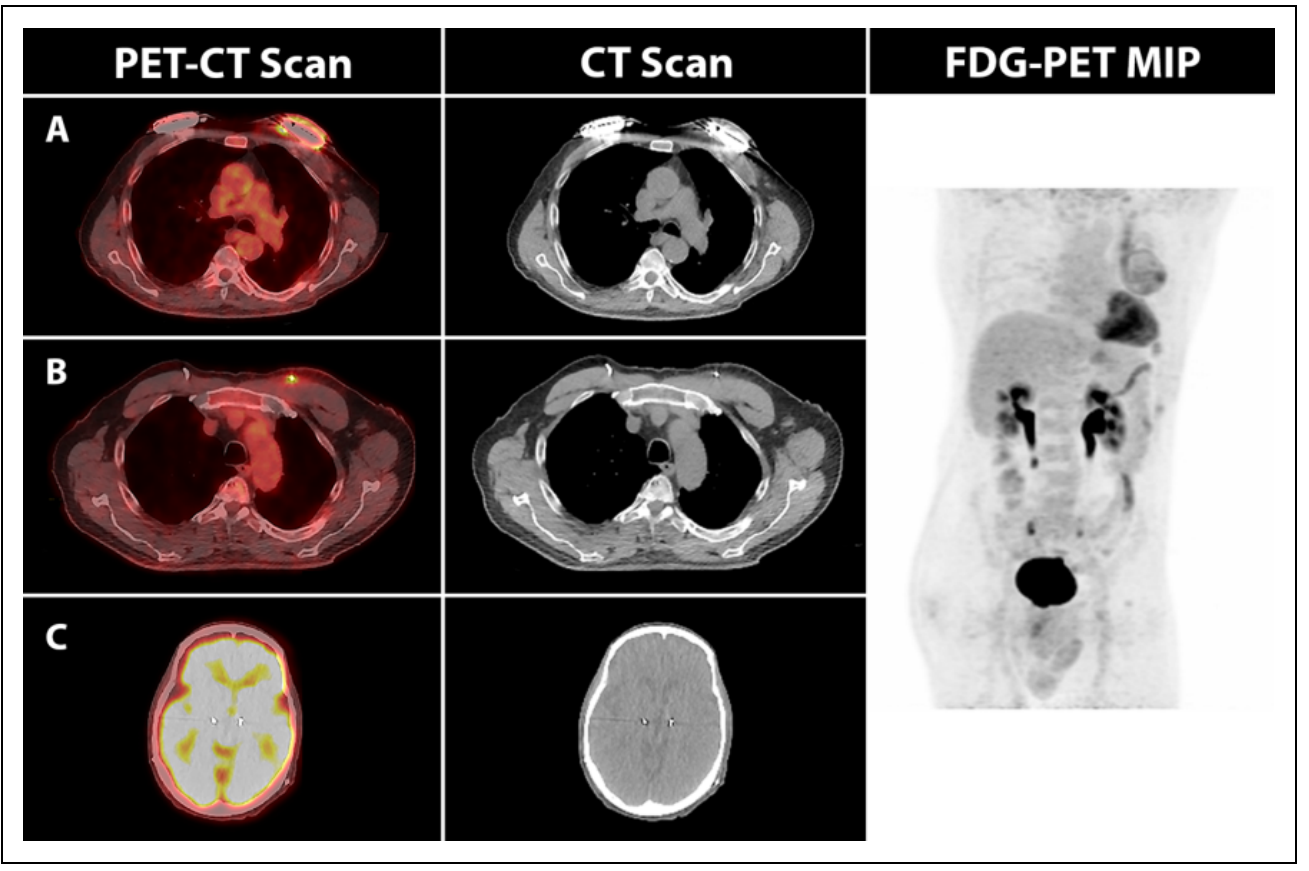

Figure I. Transaxial fused PET/CT (first column), transaxial CT (second column), and coronal maximal intensity projection FDG-PET (column 3 ) at the level of the sternum (A), aortic arch (B), and the third ventricle $(\mathrm{C})$. There is asymmetric hypermetabolic activity surrounding the left chest wall battery pack and proximal lead concerning for device infection, sparing the extracranial and intracranial portion of the lead. CT indicates computed tomography; FDG, fluorodeoxyglucose; PET, positron emission tomography.

A 71-year-old man with Parkinson disease managed with bilateral subthalamic nucleus deep brain stimulators (DBS) underwent surgical replacement of both implantable pulse generators. Ten days later, he presented to the hospital with falls and worsening of chronic dysarthria and dysphagia. He had no nuchal rigidity, photophobia, or headache. He was afebrile with a leukocytosis of $12.9 \mathrm{~K} / \mu \mathrm{L}$. Physical examination revealed erythema and edema at the left chest wall surgical site concerning for cellulitis. It was unknown whether the device was infected, and if so, to what extent.

To determine the presence or absence of device infection, fluorodeoxyglucose-positron emission tomography/computed tomography (FDG-PET/CT) was obtained (Figure 1). Hypermetabolic activity was noted around the left chest wall battery pack involving $5 \mathrm{~cm}$ of the proximal lead, concerning for device infection. The intracranial leads were well visualized and did not demonstrate any surrounding hypermetabolic activity. Computed tomography head was also obtained which was obscured by streak artifact from the DBS and not helpful in evaluating for an infectious process. The patient underwent surgical removal of the left pulse generator and extension wire

\footnotetext{
'Department of Neurology \& Neurological Sciences, Stanford University School of Medicine, Stanford, CA, USA

\section{Corresponding Author:}

Carl A. Gold, Department of Neurology \& Neurological Sciences, Stanford University School of Medicine, 300 Pasteur Drive, Room H3 I48, Stanford, CA 94305, USA.

Email: carlgold@stanford.edu
} 
and was started on empiric antibiotics. Intraoperative cultures grew Pseudomonas aeruginosa.

The FDG-PET/CT has demonstrated value in early diagnosis of cardiac device infections, including implantable cardioverter-defibrillators, pacemakers, and prosthetic valves. ${ }^{1}$ There are no published clinical guidelines regarding the diagnosis of DBS infection. Without precise diagnostic tools to demonstrate the extent of infection, the entire DBS device is often removed empirically. ${ }^{2}$ For our patient, FDG-PET/CT allowed for effective treatment without removal of the intracranial portion of the stimulator.

\section{Acknowledgments}

The authors acknowledge Sarah Cheng, MD, for her expert assistance in obtaining the high-resolution images.

\section{Declaration of Conflicting Interests}

The authors declared no potential conflicts of interest with respect to the research, authorship, and/or publication of this article.

\section{Funding}

The authors received no financial support for the research, authorship, and/or publication of this article.

\section{ORCID iD}

Shefali Dujari, MD D https://orcid.org/0000-0001-9319-6628

\section{References}

1. Chen W, Kim J, Molchanova-Cook OP, Dilsizian V. The potential of FDG PET/CT for early diagnosis of cardiac device and prosthetic value infection before morphologic damage ensue. Curr Cardiol Rep. 2014;16(3):459. doi:10.1007/s11886-013-0459-y.

2. Bjerknes S, Skogseid IM, Sæhle T, Dietrichs E, Toft M. Surgical site infections after deep brain stimulation surgery: frequency, characteristics and management in a 10-year period. Plos One. 2014;9(8):e105288. https://doi.org/10.1371/journal.pone. 0105288 . 